\title{
Anesthetics management strategy for endovascular treatment of acute ischemic stroke. A proposal for anesthetic approach
}

\author{
Emanuele Russo ${ }^{1, *}$, Daniele Bellantonio ${ }^{1}$, Marco Benni ${ }^{1}$, Emiliano Gamberini ${ }^{1}$, \\ Alessandro Circelli ${ }^{1}$, Lorenzo Viola ${ }^{1}$, Alessio Cittadini ${ }^{1}$, Luca Bissoni ${ }^{1}$, \\ Dell'Amore Cristian ${ }^{1}$, Martino Costanza ${ }^{1}$, Giuliano Bolondi ${ }^{1}$, \\ Domenico Pietro Santonastaso ${ }^{1}$, Marco Longoni ${ }^{2}$, Maria Ruggiero ${ }^{3}$, \\ Vanni Agnoletti on the behalf of the Medical Emergency Team of Ospedale Bufalini \\ Cesena $^{1}$
}

\author{
${ }^{1}$ U.O. Anestesia e Rianimazione, \\ Ospedale "M. Bufalini" AUSL della \\ Romagna Hospital, 286, Viale Ghirotti, \\ Cesena, Italy \\ ${ }^{2}$ U.O. Neurologia, Ospedale "M. Bufalini" \\ AUSL della Romagna Hospital, 286, Viale \\ Ghirotti, Cesena, Italy \\ ${ }^{3}$ U.O. Neuroradiologia, Ospedale "M. \\ Bufalini" AUSL della Romagna Hospital, \\ 286, Viale Ghirotti, Cesena, Italy

\section{*Correspondence} \\ lelegaiola@gmail.com \\ (Emanuele Russo)
}

\begin{abstract}
Acute ischemic stroke is one of the leading causes of death and long-term disability for adults.

Endovascular therapy is the standard of care for severe acute ischemic stroke, caused by large-vessel occlusion in the anterior circulation; however, the optimal anaesthetic management during the procedure is still a matter of debate. The best anesthetic treatment should mainly be related to patients' clinical conditions and the site of arterial occlusion. With this article, we share our experience based on the use of ketamine as the choosen hypnotic drug for general anesthesia, in order to avoid a sudden drop in blood pressure. The core of our proposal approach is the general anesthesia management by the medical emergency team with skills on both time-dependent diseases and neurocritical care.
\end{abstract}

\section{Keywords}

Acute ischemic stroke; Anesthesia; Blood pressure; Endovascular trombectomy; Sedation; Neurocritical care
Acute ischemic stroke (AIS) is one of the leading causes of death and long-term disability for adults [1].

Previous data report highest in-hospital mortality for cardioembolic cerebral events compared to other ischemic stroke subtypes and lower rate of absence of functional limitation at discharge [2].

In this regard, mechanical thrombectomy/endovascular treatment (EVT) has become the standard of care for managing patients with severe acute ischemic stroke caused by largevessel occlusion (LVO) in the anterior circulation [3]. An attempt to estimate neural depletion, reports that almost 1.9 million neurons can be irreversibly lost each minute before reperfusion [4].

A multidisciplinary approach is compulsory to reach optimal EVT outcome minimizing the time between stroke onset and reperfusion: it involves emergency department physicians, neurologists, interventional radiologists and anesthesiologists [5].

Optimal anesthetic management during EVT is still a matter of debate: it ranges from local anesthesia with minimal or no sedation up to general anesthesia (GA). According to the American Heart Association (AHA) guidelines [5], the early anesthetic assistance of patients with AIS should be based on individualized risk factors, neurologic status and technical evaluation of the procedure.

Clinical trial results seem to point out that benefits of an EVT carried on an immobilized patient might be undermined by impairment of physiological compensation mechanism [1].

Conscious sedation (CS) is frequently chosen as a first-line approach [6]. Conscious sedation (CS) with local anaesthesia (LA) have some potential advantages: less cardiovascular impact, shorter time to procedure start and the possibility of continuous neurologic evaluation during the procedure [7]. CS is not free from disadvantages anyway: a lack of patient immobilization and airway protection, procedural complications (i.e. longer time to reperfusion, lower grade of reperfusion, vessel perforation, embolization, etc.) $[6,7]$.

For this risk-benefit analysis, GA is considered an equally safe alternative when adequate monitoring and expertise are provided, limiting the risk of hemodynamic instability and consequent cerebral blood flow impairment $[8,9]$. While CS entails shorter time between patient arrival and femoral artery puncture, GA is likely to allow faster EVT procedures by facilitating neuroradiologists working conditions [10]. 
Optimal anesthetic treatment should mainly be related to patients' clinical conditions and the site of arterial occlusion. Patients with posterior circulation ischemia often have consciousness impairment, dysphagia and cranial nerve dysfunction [11]. For this reason, they may result to be suitable candidates for GA compared to people with anterior circulation strokes [12].

General anesthesia results to be essential in case of overwrought or aphasic patients unable to collaborate with the medical team and in case of respiratory difficulties and airways incompetence [12].

To date some important issues concerning anesthetic strategy for mechanical thrombectomy remain unsolved. The results of trials comparing CS with GA are not yet conclusive [13].

Clinical outcome of AIS, usually measured as modified Rankin Scale, deaths, hospital length of stay, depends on several variables. Trials should take into account: clinical characteristics (age, medical history, pre-stroke modified Rankin Scale NIHSS score, occlusion site), anesthetic management (drugs used, airway device, sedation monitoring depth, reductions in arterial blood pressure, $\mathrm{EtCO}_{2} / \mathrm{PaCO}_{2}$, temperature, volemia), timing (onset-to-puncture time, door-to-puncture time, time-to-reperfusion, anesthetic exposure time), radiological outcome (TICI, thrombolysis in cerebral infarction), procedural complications (parenchymal hematoma, subarachnoid hemorrhage) $[13,14]$.

This leads to the need of large sample-size for clinical trials. Caution is needed in interpreting results.

Here we report the anesthetics management strategy adopted at the Maurizio Bufalini Hospital in Cesena (Local Health Agency of Romagna subregion, Italy), a level 1 trauma center and hub center for stroke, neurosurgery and interventional radiology, serving a population of 1.200 .000 inhabitants in northern-east Italy $[15,16]$, thus resulting in about $200 \mathrm{EVT} / \mathrm{year}$ for ischemic stroke.

Acute ischemic stroke patients are primarily admitted to Bufalini Hospital by pre-hospital emergency system, or secondarily from spoke hospitals after remote informatic evaluation.

EVT is performed with the assistance of an anesthesiologist activated by in-hospital emergency system on the model of the medical emergency team (MET). Italian legislation requires the same qualification for anesthesiologist and intensive care physician.

MET consists of a senior intensive care consultant and a senior Intensive Care Unit nurse equipped with complete instrumentation to perform advanced airway management, with appropriate training to manage unstable patients [17].

It operates $24 \mathrm{~h}$ a day, 7 days a week with track and trigger activation by a phone call to the internal number " 3333 ".

In case of AIS with unstable vital parameters, MET is activated by the emergency department. Otherwise, it is systematically activated by the neuroradiologic team at the entrance of the angiographic room for diagnostic procedures or EVT. MET reaches the emergency scenario in an average time of 2.5 minutes.

Except a few selected cases, EVT is performed under GA in our institution. Our choice is to ensure patient immobilization, secure access to blood vessels for drug infusions and airway protection as fast as possible. This is why anesthesiologic management of EVT in AIS has been entrusted to the MET.

AIS patients are handled as an unstable patient: monitoring with electrocardiography (ECG), pulsoxymeter (SpO2), continuous noninvasive blood pressure (NIBP), end-tidal respiratory $\mathrm{CO} 2(\mathrm{EtCO} 2)$ and body temperature. Two peripheral vascular accesses and urinary catheter are inserted before general anesthesia induction. Rapid sequence intubation is performed with Fentanyl $100 \mathrm{mcg}$, hypnotic dose of ketamine 0.5$1 \mathrm{mg} / \mathrm{Kg} /$ predicted body weight (PBW) and succinylcholine 1 $\mathrm{mg} / \mathrm{Kg} / \mathrm{PBW}$ or Rocuronium 1,2 mg/Kg/PBW.

Inguinal puncture occurs immediately after the airway is secured.

Ketamine was chosen from our experience to avoid drop in blood pressure: in our opinion, it is an interesting option in neuro-critical care [18]. Controlled mechanical ventilation is adjusted to obtain normocapnia and avoid detrimental hypocapnia.

General anesthesia is maintained with anesthetic inhaled agents (Sevoflurane; target minimal alveolar concentration MAC 1). It is not in the aims of this paper to investigate the complex pathophysiological effects of volatile anesthetic drugs, but we have chosen halogenated gases because of their vasodilatory effect on brain circulation, useful in order to increase cerebral blood flow (CBF). Halogenated agents also decrease cerebral metabolic rate $\left(\mathrm{CMRO}_{2}\right)$ similarly to propofol [19].

If hypotension occurs over the course of EVT, target blood pressure (systolic blood pressure 140-180) [5] is preserved with norepinephrine infusion (e.v. continuous infusion 0.05 $0.2 \mu \mathrm{g} / \mathrm{kg} / \mathrm{min}$ ).

At the end of EVT, in the absence of the need for further treatment, a wake-up test is performed in the angiographic room, aiming for immediate postoperative extubation [20]. To allow a rapid neurological examination, Sugammadex (2-4 $\mathrm{mg} / \mathrm{kg}$ ) is administered under train-of-four (TOF) monitoring in case of residual curarization due to rocuronium usage before suspending anesthetic agents.

Since "Time is brain", the key point of our strategy is to consider anesthesia for EVT in AIS as an emergency procedure that must be managed by experienced consultants.

The core of our proposed approach is the management of anesthesia by medical emergency team physician; we believe that the mindset trained in time-dependent diseases and the critical care skills could be appropriate in this setting.

Anesthetic drugs and ventilatory strategy are driven by the need to avoid side effects of general anesthesia and from our internal experience.

In this paper, we have not reported any data or "magic" target number on purpose: our strategy needs to be confirmed by ongoing clinical trials.

\section{AUTHOR CONTRIBUTIONS}

Emanuele Russo, Daniele Bellantonio, Marco Benni and Emiliano Gamberini conceived the presented idea and wrote the manuscript with input and critical feedback from all authors. Alessandro Circelli, Lorenzo Viola, Alessio 
Cittadini, Luca Bissoni, Dell'Amore Cristian, Martino Costanza, Giuliano Bolondi, Domenico Pietro Santonastaso, Marco Longoni, Maria Ruggiero, Vanni Agnoletti supervised the project and critically reviewed the draft.

\section{ACKNOWLEDGMENT}

The authors thank the nursing staff for the valuable work and the high levels of professionalism.

\section{CONFLICT OF INTEREST}

The authors declare that there is no conflict of interest regarding the publication of this article.

\section{MEDICAL EMERGENCY TEAM}

1. Daniele Bellantonio

2. Beatrice Benini

3. Marco Benni

4. Giovanni Bini

5. Luca Bissoni

6. Giuliano Bolondi

7. Alessio Cittadini

8. Francesco Cocciolo

9. Benedetta De Santis

10. Cristian Dell'Amore

11. Ranieri Gaio

12. Emiliano Gamberini

13. Gasperoni Emmanuel

14. Federica Marsigli

15. Costanza Martino

16. Costantino Mastronardi

17. Manlio Cosimo Claudio Meca

18. Luca Mezzatesta

19. Alessandro Oliva

20. Silvia Olivieri

21. Piccinno Mario

22. Chiara Rosato

23. Emanuele Russo

24. Giuseppe Sabia

25. Flavia Savelli

26. Giovanni Scognamiglio

27. Andrea Sica

28. Tiziana Spada

29. Laura Tirincanti

30. Alessandra Venditto

31. Sofia Vitali

\section{DATA AVAILABILITY}

No data reported.

\section{REFERENCES}

[1] Benjamin EJ, Virani SS, Callaway CW, Chamberlain AM, Chang AR, Cheng S, et al. Heart disease and stroke statistics-2018 update: a report from the American Heart Association. Circulation. 2018; 137: e67-e492.

[2] Arboix A, Alioc J. Cardioembolic stroke: clinical features, specific cardiac disorders and prognosis. Current Cardiology Reviews. 2010; 6: $150-161$.

[3] Goyal M, Menon BK, van Zwam WH, Dippel DWJ, Mitchell PJ, Demchuk AM, et al. Endovascular thrombectomy after large-vessel ischaemic stroke: a meta-analysis of individual patient data from five randomised trials. The Lancet. 2016; 387: 1723-1731.

[4] Saver JL. Time is brain-quantified. Stroke. 2006; 37: 263-266.

[5] Powers William J, Rabinstein Alejandro A, Ackerson T, Adeoye Opeolu M, Bambakidis Nicholas C, Becker K, et al. 2018 guidelines for the early management of patients with acute ischemic stroke: a guideline for healthcare professionals from the American Heart Association/American Stroke Association. Stroke. 2018; 49: e46-e110.

[6] Ilyas A, Chen C, Ding D, Foreman PM, Buell TJ, Ironside N, et al. Endovascular mechanical thrombectomy for acute ischemic stroke under general anesthesia versus conscious sedation: a systematic review and meta-analysis. World Neurosurgery. 2018; 112: e355-e367.

[7] Rasmussen LK, Simonsen CZ, Rasmussen M. Anesthesia practice for endovascular therapy of acute ischemic stroke in Europe. Current Opinion in Anaesthesiology. 2019; 32: 523-530.

[8] Campbell BCV, van Zwam WH, Goyal M, Menon BK, Dippel DWJ, Demchuk AM, et al. Effect of general anaesthesia on functional outcome in patients with anterior circulation ischaemic stroke having endovascular thrombectomy versus standard care: a meta-analysis of individual patient data. The Lancet Neurology. 2018; 17: 47-53.

[9] Löwhagen Hendén P, Rentzos A, Karlsson J, Rosengren L, Sundeman H, Reinsfelt B, et al. Hypotension during endovascular treatment of ischemic stroke is a risk factor for poor neurological outcome. Stroke. 2015; 46: 2678-2680.

[10] van den Berg LA, Koelman DLH, Berkhemer OA, Rozeman AD, Fransen PSS, Beumer D, et al. Type of anesthesia and differences in clinical outcome after intra-arterial treatment for ischemic stroke. Stroke. 2015; 46: 1257-1262.

[11] Mattle HP, Arnold M, Lindsberg PJ, Schonewille WJ, Schroth G. Basilar artery occlusion. The Lancet Neurology. 2011; 10: 1002-1014.

[12] Hindman BJ, Dexter F. Anesthetic management of emergency endovascular thrombectomy for acute oschemic stroke, Part 2. Anesthesia \& Analgesia. 2019; 128: 706-717.

[13] Castioni CA, Rasulo F, Munari M. Letter by Castioni et al. Regarding Article, "General Anesthesia Versus Conscious Sedation and Local Anesthesia during Thrombectomy for Acute Ischemic Stroke". Stroke. 2020; 51: e331-e332.

[14] Agnoletti V, Russo E, Circelli A, Benni M, Bolondi G, Martino C, et al. From intensive care to step-down units: managing patients throughput in response to COVID-19. International Journal for Quality in Health Care. 2020. (in press)

[15] Cappellari M, Pracucci G, Forlivesi S, Saia V, Nappini S, Nencini P, et al. General anesthesia versus conscious sedation and local anesthesia during thrombectomy for acute ischemic stroke. Stroke. 2020; 51: 2036-2044.

[16] Martino C, Russo E, Santonastaso DP, Gamberini E, Bertoni S, Padovani $\mathrm{E}$, et al. Long-term outcomes in major trauma patients and correlations with the acute phase. World Journal of Emergency Surgery. 2020; 15: 6.

[17] Chieregato A, Volpi A, Gordini G, Ventura C, Barozzi M, Caspani MLR, et al. How health service delivery guides the allocation of major trauma patients in the intensive care units of the inclusive (hub and spoke) trauma system of the Emilia Romagna Region (Italy). A cross-sectional study. BMJ Open. 2017; 7: e016415.

[18] Gamberini E, Russo E, Martino C, Portolani L, Agnoletti V. "Low cost" simulation in emergency surgical airway can save lives in a level-1 Italian Major Trauma Center. Journal of Emergency Medicine and Intensive Care. 2017; 3: 117.

[19] Russo E, Santonastaso DP, Gamberini E, Circelli A, Martino C, Agnoletti V. Ketamine in neurocritical care. Journal of Intensive Care Medicine. 2020. (in press)

[20] Park S, Yook K, Yoo KY, Choi JI, Bae H, You Y, et al. Comparison of the effect of sevoflurane or propofol anesthesia on the regional cerebral oxygen saturation in patients undergoing carotid endarterectomy: a prospective, randomized controlled study. BMC Anesthesiology. 2019; 19: 157.

[21] Raming L, Moustafa H, Prakapenia A, Barlinn J, Gerber J, Theilen H, et al. Association of anesthetic exposure time with clinical outcomes after 
endovascular therapy for acute ischemic stroke. Frontiers in Neurology. 2019; 10: 679 .
How to cite this article: Emanuele Russo, Daniele Bellantonio, Marco Benni, Emiliano Gamberini, Alessandro Circelli, Lorenzo Viola. Anesthetics management strategy for endovascular treatment of acute ischemic stroke. A proposal for anesthetic approach. Signa Vitae. 2021;17(3):234-237. doi:10.22514/sv.2021.031. 